(C) The Authors 2015. This is an Open Access article, distributed under the terms of the Creative

Commons Attribution licence (http://creativecommons.org/licenses/by/4.0/), which permits unrestricted

re-use, distribution, and reproduction in any medium, provided the original work is properly cited.

\title{
No effect of modest selenium supplementation on insulin resistance in UK pregnant women, as assessed by plasma adiponectin concentration
}

\author{
Jinyuan $\mathrm{MaO}^{1,2 *}$, Sarah C. Bath ${ }^{2}$, Jessica J. Vanderlelie ${ }^{3}$, Anthony V. Perkins ${ }^{3}$, Christopher W. G. Redman ${ }^{4}$ \\ and Margaret P. Rayman ${ }^{2 *}$ \\ ${ }^{1}$ Department of Endocrinology and Metabolism, Institute of Endocrinology, The First Hospital of China Medical University, \\ Shenyang 110001, People's Republic of China \\ ${ }^{2}$ Department of Nutritional Sciences, Faculty of Health and Medical Sciences, University of Surrey, Guildford GU2 7XH, UK \\ ${ }^{3}$ School of Medical Science, Griffith Health Institute, Griffith University, Southport, QLD 4222, Australia \\ ${ }^{4}$ Nuffield Department of Obstetrics and Gynaecology, University of Oxford, Oxford OX3 9DU, UK \\ (Submitted 8 July 2015 - Final revision received 4 September 2015 - Accepted 15 September 2015 - First published online 20 October 2015)
}

\section{Abstract}

Concern has been expressed recently that Se may increase the risk of type 2 diabetes, but this has not been tested in a randomised-controlled trial (RCT) in pregnant women. We took advantage of having stored plasma samples from the Se in Pregnancy Intervention (SPRINT) RCT of Se supplementation in pregnancy to test the effect of Se supplementation on a marker of insulin resistance in UK pregnant women. Because our blood samples were not fasted, we measured plasma adiponectin concentration, a recognised marker of insulin resistance that gives valid measurements in non-fasted samples, as diurnal variability is minor and there is no noticeable effect of food intake. In SPRINT, 230 primiparous UK women were randomised to treatment with Se $(60 \mu \mathrm{g} / \mathrm{d})$ or placebo from 12 weeks of gestation until delivery. We hypothesised that supplementation with Se at a nutritional level would not exacerbate the fall in adiponectin concentration that occurs in normal pregnancy, indicating the lack of an adverse effect on insulin resistance. Indeed, there was no significant difference between the two groups in the change in adiponectin from 12 to 35 weeks $(P=0.938)$, nor when the analysis was restricted to the bottom or top quartiles of baseline whole-blood Se $(P=0.515$ and 0.858 , respectively). Cross-sectionally, adiponectin concentration was not associated with any parameter of Se status, either at 12 or 35 weeks. It is reassuring that a nutritional dose of Se had no adverse effect on the concentration of adiponectin, a biomarker of insulin resistance, in pregnant women of modest Se status.

\section{Key words: Selenium: Pregnancy: Adiponectin: Diabetes: Insulin resistance}

Concern has been expressed in recent years that Se may increase the risk of type 2 diabetes (T2D), and indeed a number of studies have implicated the selenoproteins, cytosolic glutathione peroxidase 1 (GPx1) and selenoprotein P1 (SEPP1) in insulin resistance $^{(1-4)}$. However, epidemiological data are conflicting. Although higher Se status has been associated with higher prevalence of T2D or fasting plasma glucose in five cross-sectional studies, three such studies have found the opposite association and two have found no association ${ }^{(1,5-7)}$. Results of longitudinal or prospective studies have not supported a causal role for Se in T2 $\mathrm{D}^{(1)}$. With regard to trial evidence, five randomised-controlled trials (RCT) with Se as a single agent reported on the risk of T2D, although none had T2D as a primary end point ${ }^{(1,8,9)}$. Of these, only one showed an increased incidence of T2D in the Se arm, although only in participants in the top tertile of plasma Se at baseline ${ }^{(10)}$.

Studies on pregnant women are also contradictory; in pregnant women without gestational diabetes (GDM), activity of the cytosolic selenoenzyme, GPx1, in erythrocytes increased during pregnancy and was positively associated with fasting plasma glucose, plasma insulin, C-peptide and the homoeostasis model of assessment for insulin resistance (HOMA-IR) index, suggesting an adverse effect of Se, or at least of a selenoprotein ${ }^{(11)}$. By contrast, the literature also shows that pregnant women with impaired glucose tolerance or GDM have much lower serum Se concentrations than women with normal pregnancies $^{(12-14)}$ and that there is an inverse relationship between serum Se and blood glucose concentration ${ }^{(14)}$. Furthermore, in a small group of pregnant women, the increase in plasma glucose following an oral glucose tolerance test administered at 12 weeks of gestation was inversely correlated with plasma Se concentration ${ }^{(15)}$. Recently, an RCT of Se $(200 \mu \mathrm{g} / \mathrm{d})$ in seventy women who had already developed GDM found a significant reduction in fasting plasma glucose, serum insulin and HOMA-IR in those on $\mathrm{Se}^{(16)}$. However, to date, there

Abbreviations: GDM, gestational diabetes; GPx3, glutathione peroxidise 3; RCT, randomised-controlled trial; SEPP1, selenoprotein P1; T2D, type 2 diabetes. 
has been no RCT of the effect of Se supplementation on the risk of GDM or insulin resistance in normal pregnant women.

We took advantage of having stored plasma samples from the Se in Pregnancy Intervention (SPRINT) RCT of Se supplementation in pregnancy to test the effect of Se supplementation on a marker of insulin resistance in UK pregnant women ${ }^{(17)}$. SPRINT was set up to assess the effect of Se supplementation on the risk of pre-eclampsia in pregnant women of low-to-moderate Se status ${ }^{(17)}$. Although we could not validly measure plasma glucose or insulin as we only had non-fasted samples, we were able to measure plasma adiponectin, a recognised marker of insulin resistance $^{(18)}$ and a strong independent predictor of the risk of GDM and $\mathrm{T} 2 \mathrm{D}^{(19-24)}$. Adiponectin measurements are valid even in non-fasted plasma samples, as diurnal variability is minor and there is no noticeable effect of food intake $e^{(19,20,25,26)}$. Higher plasma adiponectin concentration is associated with lower risk ${ }^{(20)}$.

Plasma adiponectin concentration falls substantially during the course of normal pregnancy ${ }^{(27)}$. We hypothesised that supplementation with Se at a nutritional level would not exacerbate that fall, indicating a lack of an adverse effect of Se on insulin resistance. On the basis of our previous findings ${ }^{(17)}$, we speculated that Se supplementation might even lessen the fall in plasma adiponectin in women in the bottom quartile of Se status at baseline.

\section{Methods \\ Participants}

Blood and plasma samples for this study originate from the SPRINT study (trial registration ISRCTN37927591, http://controlledtrials. com/ISRCTN37927591), which randomised 230 primiparous women in Oxford, UK, to treatment with Se $(60 \mu \mathrm{g} / \mathrm{d}$ Se, as Se yeast) or placebo (placebo yeast) from their first hospital antenatal visit (mean gestational age 12.3 weeks) until delivery of the baby ${ }^{(17)}$. Blood samples, from which plasma was prepared, were collected at baseline (12 weeks), 20 and 35 weeks ${ }^{(17)}$.

Five women were excluded from the analysis: one woman was recruited in error (treated with thyroxine), two had diabetes before conception and two were outliers, one with respect to wholeblood Se and one with respect to adiponectin concentration. One woman had no remaining plasma for adiponectin analysis at 12 weeks. There were 111 women in the placebo group and 113 in the Se group at baseline, and 107 in the placebo group and 104 in the Se group at 35 weeks (see Participant Flow chart in previous publication ${ }^{(17)}$ ).

The study was conducted in accordance with the guidelines of the Declaration of Helsinki. All procedures involving human subjects were approved by the Milton Keynes Research Ethics Committee (REC reference no. 08/H0603/46). A non-substantial amendment for additional laboratory measurements in stored samples was approved by NRES Committee South Central, Berkshire (27 July 2011). Written informed consent was obtained from all subjects.

\section{Anthropometric measurements}

Weight at 12 and 35 weeks was measured to the nearest $0 \cdot 1 \mathrm{~kg}$ while subjects were dressed in light clothing. Subjects stood barefoot while height was measured to the nearest $0.01 \mathrm{~m}$ by using a wall-mounted stadiometer (Holtain Ltd). BMI was calculated using weight $(\mathrm{kg})$ divided by height squared $\left(\mathrm{m}^{2}\right)$.

\section{Laboratory analyses}

Whole-blood Se and plasma glutathione peroxidase 3 (GPx3) activity at 12 and 35 weeks were determined by inductively coupled plasma MS and a spectrophotometric assay, respectively, as previously described ${ }^{(17,28)}$. The CV for the whole-blood Se assay was $0.25 \%$ at $1.4 \mathrm{mmol} / 1$ and $0.17 \%$ at $3.0 \mathrm{mmol} / \mathrm{l}^{(28)}$. For GPx3, the inter-assay variation was $0.43 \%$ and the intra-assay variation was $1.25 \%^{(29)}$

Toenail Se at 16 weeks was measured using instrumental neutron activation analysis at the Interfaculty Reactor Institute in Delft, as previously described ${ }^{(17,30)}$. The laboratory has an embedded quality-control system for quality assurance and management, which complies with the requirements of the International Standard ISO/IEC 17025:2005 and has been accredited by the Dutch Council for Accreditation since 1993.

Plasma SEPP1 concentration at 35 weeks was measured by ELISA in the laboratory of Raymond Burk, as previously described $^{(17,30)}$. For quality control, each ELISA was run with two standards: purified SEPP1 and a standard plasma sample ${ }^{(31)}$

Total plasma adiponectin concentration at 12 and 35 weeks was measured using a commercial ELISA kit (Quantikine ${ }^{\circledR}$, Human Total Adiponectin/Acrp30 Immunoassay; R\&D Systems Europe Ltd) according to the manufacturer's instructions (http://www.rndsystems.com/pdf/drp300.pdf). The intra- and inter-assay CV were 2.5 and $6.8 \%$, respectively.

\section{Statistical methods}

To use parametric analysis, adiponectin concentration was transformed using the natural logarithm (ln) to approximate a normal distribution. Then, the difference at baseline between placebo and Se groups was tested by an independent samples $t$ test. A paired samples $t$ test was used to compare adiponectin concentrations at 12 and 35 weeks within placebo and Se groups.

Intention-to-treat analysis. The difference in the change in adiponectin between placebo and Se groups from 12 to 35 weeks was tested by the Mann-Whitney $U$ test. On the basis of effects seen previously in this study in women in the bottom Se status quartile (whole-blood Se) ${ }^{(17)}$, we repeated the test in the group of women in the bottom quartile of whole-blood Se at baseline. Because a previous study saw adverse effects only in those in the top tertile of baseline plasma $\mathrm{Se}^{(10)}$, we also carried out the same analysis in those of high status - that is, women in the top quartile in the current study. We also explored the difference in adiponectin concentration between placebo and Se groups at 35 weeks by ANCOVA, by adjusting for baseline ln-adiponectin concentration, as adiponectin concentration at baseline and 35 weeks were highly correlated $(r 0.755 ; P<0.001)$.

For the sample size per treatment group and an uncorrected two-sided significance level of 0.05 , the trial had $80 \%$ power to detect a $25.1 \%$ difference in adiponectin change during pregnancy between Se and placebo groups. 
Cross-sectional associations. To evaluate the cross-sectional associations between Se status and adiponectin concentration at baseline ( 12 weeks), we divided the population into quartiles by the various Se status parameters. The median value of each quartile was used as an ordinal variable to test the significance of the linear trend across quartiles by multiple linear regression analysis. Adjustment was made for baseline BMI, as BMI is known to be associated with adiponectin concentration ${ }^{(32)}$ as was also the case in our study. No adjustment was made for gestational age despite the fact that it affects adiponectin concentration ${ }^{(27)}$, as the range of gestational ages was very narrow (median 12 (interquartile range (IQR) 12, 13) weeks).

As the magnitude and range of both Se status and adiponectin concentration changed substantially over the course of the trial, we carried out a cross-sectional analysis at 35 weeks, stratified by treatment group. In-transformed adiponectin concentration at 35 weeks was adjusted for baseline ln-transformed adiponectin and BMI at 35 weeks.

Statistical analyses were conducted using IBM SPSS Statistics version 20. $P$ values $<0.05$ (two-tailed) were considered significant.

\section{Results}

\section{Baseline characteristics}

Table 1 shows the baseline adiponectin concentrations in the group overall and in Se and placebo groups separately, and the $P$ value for comparison between the groups. There was no significant difference in adiponectin concentration between treatment groups at baseline. We previously reported the lack of any significant difference in parameters of Se status (whole-blood Se, GPx3 activity, toenail Se) and other participant characteristics (age, ethnicity, gestational age, smoking, drinking, BMI) between treatment groups at baseline ${ }^{(17,28)}$.

\section{Effect of treatment}

At 35 weeks, median whole-blood Se in the Se group was significantly higher than in the placebo group (1.87 (IQR 1.67, 2.15) v. 1.16 (IQR $1.05,1.30) \mu \mathrm{mol} / \mathrm{l} ; P<0.001)$, although there was no significant difference in GPx3 activity between the two groups $(P=0 \cdot 140)$. Compared with baseline, in the women

Table 1. Baseline adiponectin concentrations in the group overall and in selenium and placebo groups separately, and $P$ value for comparison between groups (Medians and interquartile ranges (IQR); geometric means and $95 \%$ confidence intervals)

\begin{tabular}{lccc}
\hline Adiponectin concentrations $(\mathrm{mg} / \mathrm{l})$ & Overall & Se group & Placebo group \\
\hline Number of women & 224 & 113 & 111 \\
Median & $12 \cdot 51$ & $12 \cdot 90$ & $11 \cdot 11$ \\
IQR & $8 \cdot 90,16 \cdot 54$ & $9 \cdot 69,16 \cdot 54$ & $7 \cdot 93,16 \cdot 57$ \\
Mean & $11 \cdot 76$ & $12 \cdot 37$ & $11 \cdot 18$ \\
$95 \% \mathrm{Cl}$ & $11 \cdot 10,12.47$ & $11 \cdot 39,13.41$ & $10 \cdot 30,12 \cdot 13$ \\
\hline
\end{tabular}

${ }^{*} P$ value for difference between Se and placebo groups by independent samples $t$ test.

Table 2. Effect of selenium supplementation on change in plasma adiponectin concentration between 12 and 35 weeks of gestation in women with samples at both 12 and 35 weeks, in all participants and in the bottom and top quartiles of whole-blood selenium (whole group) at baseline (Geometric means and $95 \%$ confidence intervals; medians and interquartile ranges (IQR))

\begin{tabular}{|c|c|c|c|c|c|}
\hline \multirow[b]{2}{*}{ Participants included } & \multicolumn{2}{|c|}{ Se group } & \multicolumn{2}{|c|}{ Placebo group } & \multirow[b]{2}{*}{$P^{*}$} \\
\hline & Mean & $95 \% \mathrm{Cl}$ & Mean & $95 \% \mathrm{Cl}$ & \\
\hline All participants $(n)$ & \multicolumn{2}{|c|}{104} & \multicolumn{2}{|c|}{106} & \\
\hline 12-week adiponectin concentration (mg/l) & $12 \cdot 55$ & $11.54,13.64$ & $11 \cdot 21$ & $10 \cdot 26,12 \cdot 25$ & \\
\hline 35-week adiponectin concentration (mg/l) & 9.50 & $8.62,10.48$ & $8 \cdot 30$ & $7.59,9.08$ & \\
\hline $\mathrm{P}+$ & \multicolumn{2}{|c|}{$<0.001$} & \multicolumn{2}{|c|}{$<0.001$} & \\
\hline Change in adiponectin concentration (mg/l) & & & & & 0.938 \\
\hline Median & \multicolumn{2}{|c|}{-2.91} & \multicolumn{2}{|c|}{-2.79} & \\
\hline IQR & \multicolumn{2}{|c|}{$-4.80,-0.43$} & \multicolumn{2}{|c|}{$-5 \cdot 16,-1.09$} & \\
\hline Bottom quartile of whole-blood Se $(n)$ & \multicolumn{2}{|c|}{25} & \multicolumn{2}{|c|}{29} & \\
\hline 12-week adiponectin concentration (mg/l) & $12 \cdot 25$ & $10 \cdot 52,14 \cdot 27$ & $10 \cdot 52$ & $8 \cdot 72,12 \cdot 70$ & \\
\hline 35-week adiponectin concentration (mg/l) & 9.36 & $7.91,11.08$ & 8.28 & $6.81,10.07$ & \\
\hline$P+$ & \multicolumn{2}{|c|}{$<0.001$} & \multicolumn{2}{|c|}{$<0.001$} & \\
\hline Change in adiponectin concentration (mg/l) & & & & & 0.515 \\
\hline Median & \multicolumn{2}{|c|}{$-2 \cdot 80$} & \multicolumn{2}{|c|}{-2.42} & \\
\hline IQR & \multicolumn{2}{|c|}{$-5 \cdot 23,-0.26$} & \multicolumn{2}{|c|}{$-3.70,0.03$} & \\
\hline Top quartile of whole-blood Se $(n)$ & \multicolumn{2}{|c|}{24} & \multicolumn{2}{|c|}{29} & \\
\hline 12-week adiponectin concentration (mg/l) & 13.14 & $11.41,15.50$ & $12 \cdot 59$ & $10 \cdot 61,14.95$ & \\
\hline 35-week adiponectin concentration (mg/l) & 8.92 & $7 \cdot 20,11.05$ & 8.50 & $7 \cdot 10,10 \cdot 17$ & \\
\hline $\mathrm{P}+$ & \multicolumn{2}{|c|}{$<0.001$} & \multicolumn{2}{|c|}{$<0.001$} & \\
\hline Change in adiponectin concentration (mg/l) & & \multirow{2}{*}{\multicolumn{2}{|c|}{-3.89}} & $0 \cdot 858$ \\
\hline Median & & & & & \\
\hline IQR & \multicolumn{2}{|c|}{$-6 \cdot 18,-1 \cdot 78$} & \multicolumn{2}{|c|}{$-6.93,-0.90$} & \\
\hline
\end{tabular}

* $P$ value for comparison between Se and placebo treatment groups for median change in adiponectin by Mann-Whitney $U$ test.

$\dagger P$ value from paired $t$ test, $12 v$. 35 weeks. 
Effect of selenium on adiponectin in pregnancy

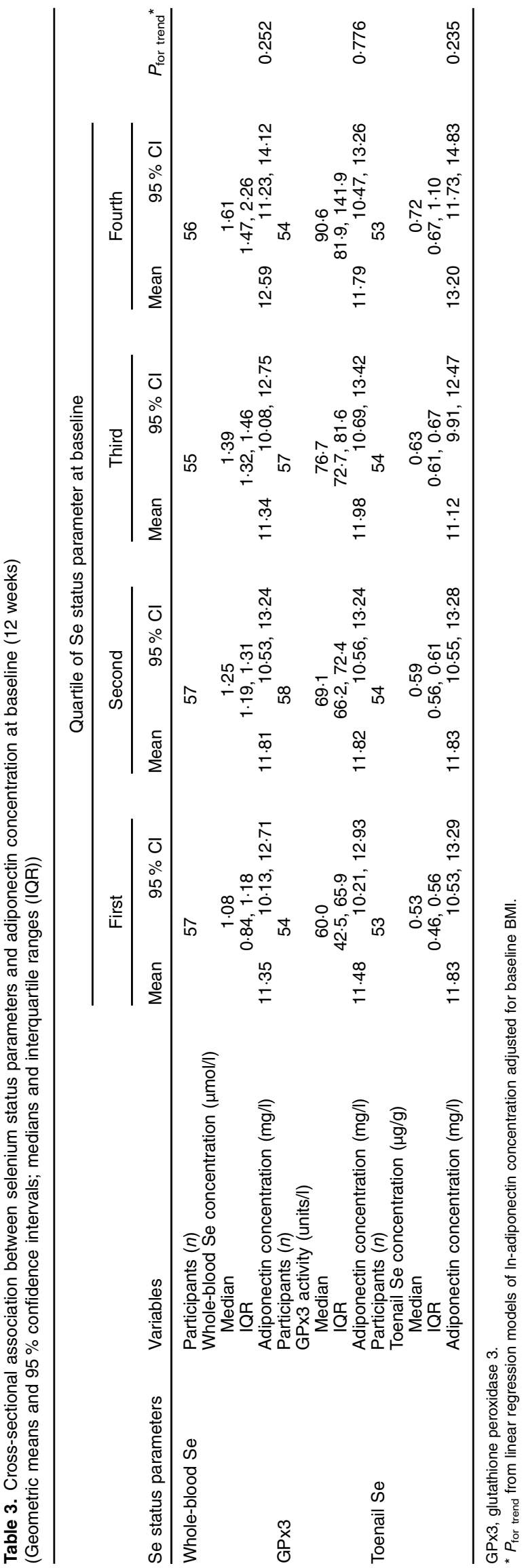

supplemented with Se, whole-blood Se and GPx3 activity at 35 weeks significantly increased by $42.1 \%(P<0.001)$ and $6.7 \%$ ( $P=0.026$ ), respectively. In the placebo group, between baseline and 35 weeks, whole-blood Se significantly decreased by $12.8 \%(P<0.001)$ but GPx3 activity did not change $(P=0.874)$.

\section{Intention-to-treat analysis}

Adiponectin concentration significantly decreased during pregnancy in both placebo and Se groups, by 23.7 and $25.5 \%$, respectively $(P<0.001$ for both). However, there was no significant difference in the change in adiponectin during pregnancy between the two groups $(P=0.938)$, nor when the analysis was restricted to women in the bottom or top quartile of whole-blood Se at baseline (Table 2). At 35 weeks, adiponectin concentration was highly and significantly correlated with that at baseline in the overall population ( $r$ 0.755; $P<0.001$ ). When 35-week adiponectin concentration was adjusted for baseline concentration, ANCOVA found no significant difference in adiponectin concentration between placebo and Se groups $(P=0 \cdot 332)$.

Two women (1.8\%) in the placebo group were diagnosed with GDM in late pregnancy but none in the Se group; however, clearly no significance can be attached to this finding.

\section{Cross-sectional association between parameters of selenium status and adiponectin concentration at baseline}

To investigate cross-sectional association between parameters of Se status and adiponectin concentration, we divided participants by quartile of Se status at baseline. Adiponectin concentration was not associated with any parameter of Se status (Table 3). Although the geometric mean of plasma adiponectin was higher in the top than in the bottom quartile of Se status for whole-blood Se, GPx3 activity and toenail Se, the difference did not reach significance (adjusted $P_{\text {for linear trend }}=0.252,0.776$ and 0.235 , respectively).

\section{Cross-sectional association between adiponectin concentrations and selenium status parameters in the placebo group at 35 weeks}

As adiponectin concentration fell substantially from 12 to 35 weeks, we explored whether there might be a cross-sectional association with Se status at that lower level of adiponectin that represents a higher level of insulin resistance or risk of GDM. We therefore carried out a cross-sectional analysis of the effect of 35-week Se status parameters on 35-week baseline-adjusted In-adiponectin concentration in each group separately. Although there was a nearsignificant positive association of GPx3 activity with adiponectin concentration in both placebo and Se groups, it did not reach significance $(P=0.061$ and $0 \cdot 077$, respectively, Table 4). None of the other Se status parameters was associated with adiponectin concentration.

\section{Discussion}

The lack of a difference between the effect of Se and placebo supplementation on the change in plasma adiponectin 


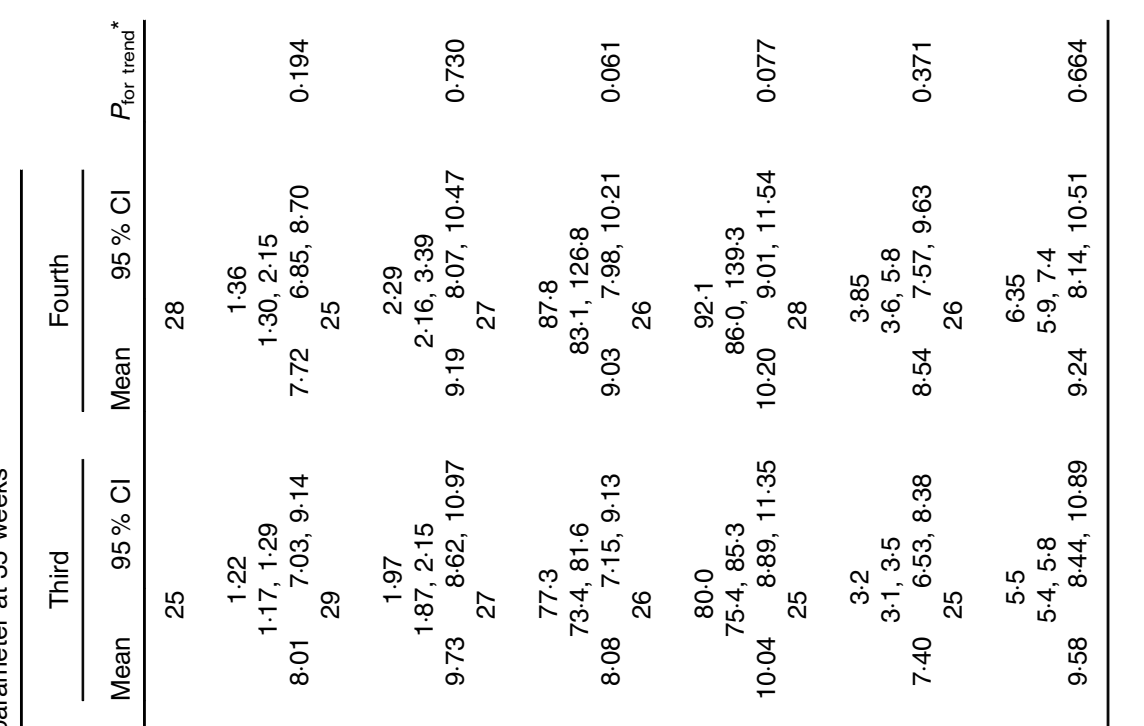

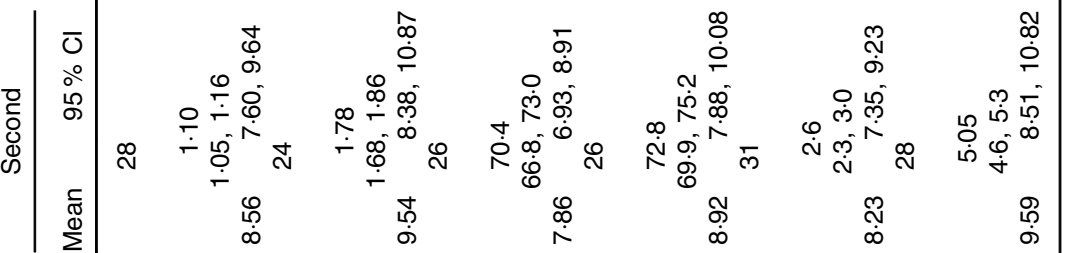

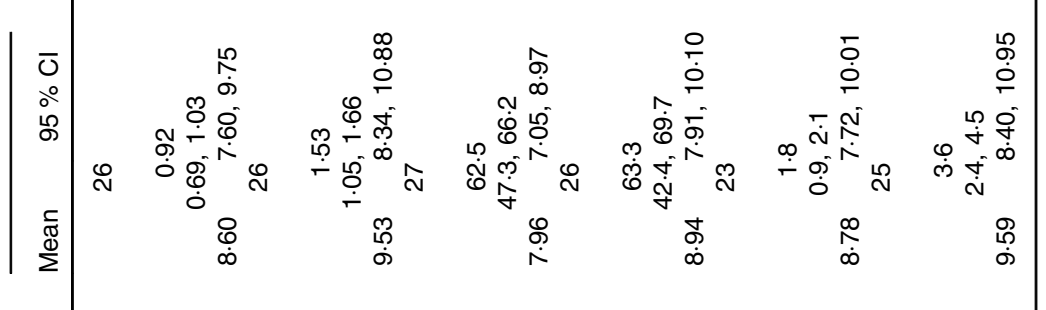

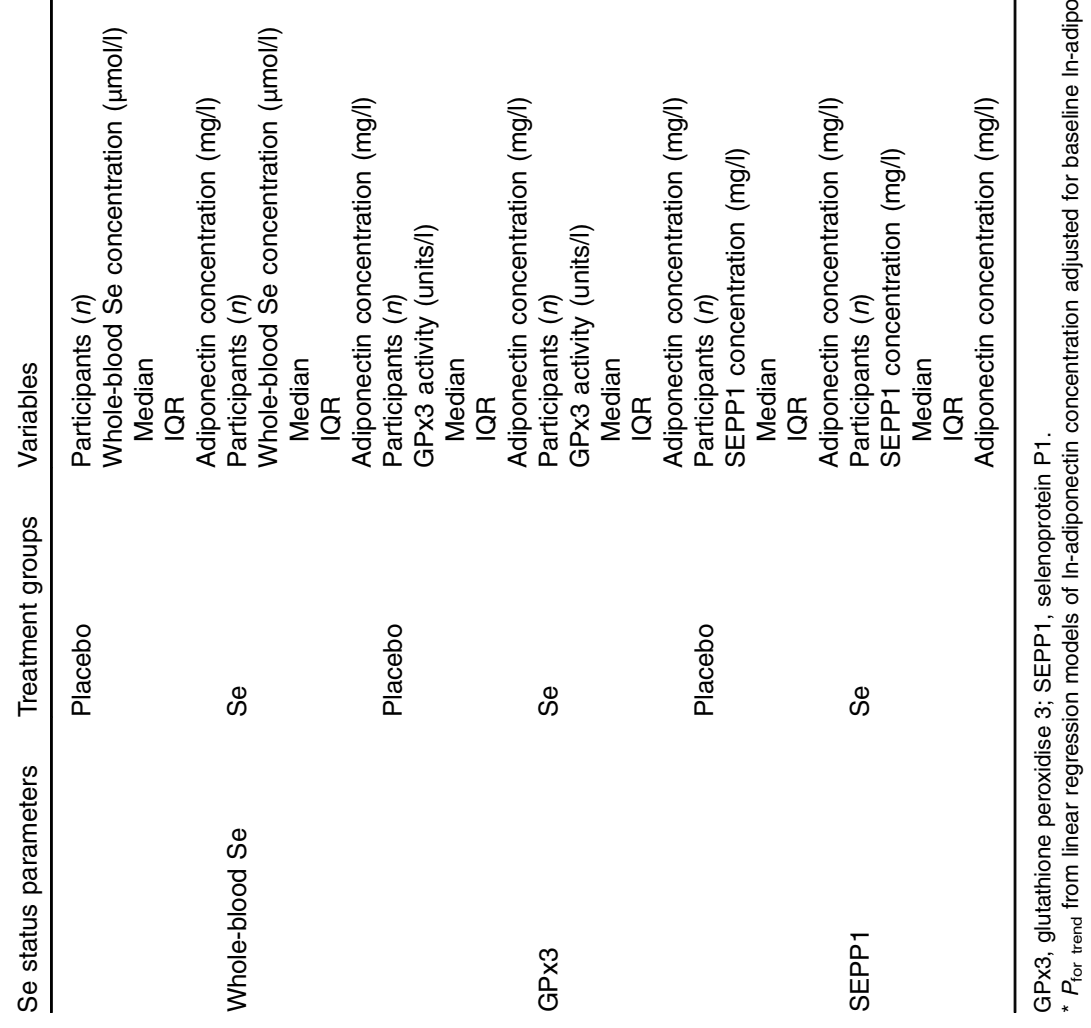


concentration from 12 to 35 weeks $(P=0.938)$ confirmed our hypothesis that supplementation with Se at a nutritional level would not adversely affect plasma adiponectin. Although we had speculated that Se supplementation to women in the bottom quartile of Se status at baseline might benefit them by reducing the fall in plasma adiponectin, we did not see any such benefit $(P=0.515)$.

The significant fall that we observed in plasma adiponectin from 12 to 35 weeks gestation is in line with previous findings ${ }^{(27)}$ and corresponds to an impairment of peripheral insulin sensitivity in late pregnancy that allows increased glucose availability to the feto-placental unit ${ }^{(27,33)}$.

Taking plasma adiponectin as a proxy for insulin resistance ${ }^{(18)}$, our results are reassuring insofar as they demonstrate the lack of an adverse effect of low-dose Se on insulin resistance in pregnancy, at least in a pregnant population of relatively low Se status such as that of the $\mathrm{UK}^{(34)}$. The only other RCT that used plasma adiponectin as biomarker of insulin resistance/T2D similarly found no diabetogenic effect, despite the Se dose levels being considerably higher, i.e., 100,200 and $300 \mu \mathrm{g} / \mathrm{d}^{(9)}$; that trial was also carried out in a UK population. In fact, the only trial that has found an increased risk of T2D was in a US population of considerably higher Se status than that of the UK, and it gave quite a substantial dose of Se, i.e., $200 \mu \mathrm{g} / \mathrm{d}^{(10)}$.

We divided the various parameters of Se status into quartiles to see whether we could identify any cross-sectional associations at baseline, as had been seen in the previous trial of Se with plasma adiponectin as an outcome ${ }^{(9)}$. Although the geometric mean of plasma adiponectin was higher in the top than in the bottom quartile for all Se status parameters, the difference did not reach significance.

No cross-sectional association was found between 35-week SEPP1 and plasma adiponectin, although an association had been found previously between serum adiponectin and SEPP1; however, that association was in patients with $\mathrm{T}_{2} \mathrm{D}^{(35)}$. It is, nonetheless, of interest that we found a near-significant positive correlation of GPx3 activity with adiponectin concentration in both placebo and Se groups at 35 weeks, a time when adiponectin concentration had fallen substantially. GPx3 concentration has been found to be significantly lower in women with T2D than in those with normal glucose tolerance, and GPx3 expression has been shown to be down-regulated in T2D muscle ${ }^{(36)}$. Furthermore, the ability of the thiazolidinediones to prevent insulin resistance induced by oxidative stress in human skeletal muscle cells is exclusively mediated by GPx3 ${ }^{(36)}$. We may have been limited by our study size in not seeing a significant correlation with GPx3. It may be relevant that the near-significant effect of GPx3 that we saw appears to be independent of the effect of Se, per se. This may suggest that an SNP in the GPX3 gene affects adiponectin concentration in pregnancy. We are currently investigating such a potential GPX3 genotype effect.

Our study was limited by being a secondary analysis of a trial that was set up for another purpose, and hence our sample size would not have been adequate to detect a small treatment effect. A further limitation was that we only had one marker of insulin resistance owing to a lack of fasted samples; however, adiponectin is a well-recognised marker of insulin resistance $^{(18)}$ and it has been shown to be an independent predictor of the risk of $\mathrm{GDM}^{(23,24)}$.

In conclusion, a nutritional dose of Se given to pregnant women of modest Se status in an RCT had no apparent adverse effect on the concentration of adiponectin, a biomarker of insulin resistance, nor did there appear to be a beneficial effect, although some previous studies might have predicted benefit ${ }^{(12-16)}$; however, the dose was small. Given the known positive associations between higher Se status or Se supplementation and healthy pregnancy, this is good news for women in countries such as the UK and Europe who are unlikely to increase the risk of insulin resistance by taking a modest Se supplement ${ }^{(15,17,28,30,37-39)}$.

\section{Acknowledgements}

The authors are extremely grateful to all the women who took part in this study, and the team at the John Radcliffe Hospital, especially research midwife, Libby Searle, who recruited the women. We thank Christine Sieniawska of the Trace Element Unit, Southampton University Hospital NHS Trust for analysis of Se in whole blood and Mehmet Sarilar and Menno Blaauw of TU Delft Reactor Instituut who analysed the toenails.

The SPRINT study was supported by a Wellcome Trust Project grant no. 083918/Z/07/Z (M. P. R., C. W. G. R.); adiponectin measurement was supported by Nestle Nutrition Institute Fellowship; J. M. was supported by Shenyang Science and Technology Project (F12-193-9-28). S. C. B. was supported by an MRC Population Health Scientist Fellowship. Toenail analysis at TU Delft Reactor Instituut was supported by the European Commission under the 7th Framework Programme through the 'Research Infrastructures' action of the 'Capacities' Programme (NMI3-II grant no. 283883). The funders had no role in the design, analysis or writing of this article.

M. P. R. was the SPRINT study principal investigator and C. W. G. R. was responsible for the recruitment of the women at the John Radcliffe Hospital. M. P. R. and J. M. designed the current research project. J. J. V. and A. V. P. were responsible for measuring GPx3 activity. J. M. performed adiponectin measurement and performed the statistical analysis. S. C. B. advised on the data analysis. M. P. R. wrote the paper with help from J. M., M. P. R. had primary responsibility for final content. All authors read and approved the final manuscript.

There are no conflicts of interest.

\section{References}

1. Rayman MP \& Stranges S (2013) Epidemiology of selenium and type 2 diabetes: can we make sense of it? Free Radic Biol Med 65, 1557-1564.

2. Misu H, Takamura T, Takayama H, et al. (2010) A liver-derived secretory protein, selenoprotein $\mathrm{P}$, causes insulin resistance. Cell Metab 12, 483-495.

3. Yang SJ, Hwang SY, Choi HY, et al. (2011) Serum selenoprotein $\mathrm{P}$ levels in patients with type 2 diabetes and prediabetes: implications for insulin resistance, inflammation, and atherosclerosis. J Clin Endocrinol Metab 96, E1325-E1329.

4. Hellwege JN, Palmer ND, Ziegler JT, et al. (2014) Genetic variants in selenoprotein $\mathrm{P}$ plasma 1 gene (SEPP1) are associated with fasting insulin and first phase insulin response in Hispanics. Gene 534, 33-39. 
5. Navarro-Alarcon M, Lopez G, Perez-Valero V, et al. (1999) Serum and urine selenium concentrations as indicators of body status in patients with diabetes mellitus. Sci Total Environ 228, 79-85.

6. Kljai K \& Runje R (2001) Selenium and glycogen levels in diabetic patients. Biol Trace Elem Res 83, 223-229.

7. Rajpathak S, Rimm E, Morris JS, et al. (2005) Toenail selenium and cardiovascular disease in men with diabetes. $J$ Am Coll Nutr 24, 250-256.

8. Mao S, Zhang A \& Huang S (2014) Selenium supplementation and the risk of type 2 diabetes mellitus: a meta-analysis of randomized controlled trials. Endocrine 47, 758-763.

9. Rayman MP, Blundell-Pound G, Pastor-Barriuso R, et al. (2012) A randomized trial of selenium supplementation and risk of type- 2 diabetes, as assessed by plasma adiponectin. PLOS ONE 7, e45269.

10. Stranges S, Marshall JR, Natarajan R, et al. (2007) Effects of long-term selenium supplementation on the incidence of type 2 diabetes: a randomized trial. Ann Intern Med $\mathbf{1 4 7}$ $217-223$.

11. Chen X, Scholl TO, Leskiw MJ, et al. (2003) Association of glutathione peroxidase activity with insulin resistance and dietary fat intake during normal pregnancy. J Clin Endocrinol Metab 88, 5963-5968.

12. Tan M, Sheng L, Qian Y, et al. (2001) Changes of serum selenium in pregnant women with gestational diabetes mellitus. Biol Trace Elem Res 83, 231-237.

13. Al-Saleh E, Nandakumaran M, Al-Shammari M, et al. (2004) Maternal-fetal status of copper, iron, molybdenum, selenium and zinc in patients with gestational diabetes. J Matern Fetal Neonatal Med 16, 15-21.

14. Kilinc M, Guven MA, Ezer M, et al. (2008) Evaluation of serum selenium levels in Turkish women with gestational diabetes mellitus, glucose intolerants, and normal controls. Biol Trace Elem Res 123, 35-40.

15. Hawkes WC, Alkan Z, Lang K, et al. (2004) Plasma selenium decrease during pregnancy is associated with glucose intolerance. Biol Trace Elem Res 100, 19-29.

16. Asemi Z, Jamilian M, Mesdaghinia E, et al. (2015) Effects of selenium supplementation on glucose homeostasis, inflammation, and oxidative stress in gestational diabetes: randomized, double-blind, placebo-controlled trial. Nutrition 31, 1235-1242.

17. Rayman MP, Searle E, Kelly L, et al. (2014) Effect of selenium on markers of risk of pre-eclampsia in UK pregnant women: a randomised, controlled pilot trial. Br J Nutr 112, 99-111.

18. Kishida K, Funahashi T \& Shimomura I (2012) Molecular mechanisms of diabetes and atherosclerosis: role of adiponectin. Endocr Metab Immune Disord Drug Targets 12, 118-131.

19. Hotta K, Funahashi T, Arita Y, et al. (2000) Plasma concentrations of a novel, adipose-specific protein, adiponectin, in type 2 diabetic patients. Arterioscler Thromb Vasc Biol 20, $1595-1599$.

20. Li S, Shin HJ, Ding EL, et al. (2009) Adiponectin levels and risk of type 2 diabetes: a systematic review and meta-analysis. JAMA 302, 179-188.

21. Thorand B, Zierer A, Baumert J, et al. (2010) Associations between leptin and the leptin/adiponectin ratio and incident type 2 diabetes in middle-aged men and women: results from the MONICA/KORA Augsburg study 1984-2002. Diabet Med 27, 1004-1011.
22. Montonen J, Drogan D, Joost HG, et al. (2011) Estimation of the contribution of biomarkers of different metabolic pathways to risk of type 2 diabetes. Eur J Epidemiol 26, 29-38.

23. Lacroix M, Battista MC, Doyon M, et al. (2013) Lower adiponectin levels at first trimester of pregnancy are associated with increased insulin resistance and higher risk of developing gestational diabetes mellitus. Diabetes Care 36, 1577-1583.

24. Fasshauer M, Bluher M \& Stumvoll M (2014) Adipokines in gestational diabetes. Lancet Diabetes Endocrinol 2, 488-499.

25. Gavrila A, Peng CK, Chan JL, et al. (2003) Diurnal and ultradian dynamics of serum adiponectin in healthy men: comparison with leptin, circulating soluble leptin receptor, and cortisol patterns. J Clin Endocrinol Metab 88, 2838-2843.

26. Shand B, Elder P, Scott R, et al. (2006) Biovariability of plasma adiponectin. Clin Chem Lab Med 44, 1264-1268.

27. Catalano PM, Hoegh M, Minium J, et al. (2006) Adiponectin in human pregnancy: implications for regulation of glucose and lipid metabolism. Diabetologia 49, 1677-1685.

28. Rayman MP, Bath SC, Westaway J, et al. (2015) Selenium status in UK pregnant women and its relationship with hypertensive conditions of pregnancy. Br J Nutr (epublication ahead of print version 9 January 2015).

29. Vanderlelie J, Venardos K, Clifton VL, et al. (2005) Increased biological oxidation and reduced anti-oxidant enzyme activity in pre-eclamptic placentae. Placenta 26, 53-58.

30. Rayman MP, Bode P \& Redman CW (2003) Low selenium status is associated with the occurrence of the pregnancy disease preeclampsia in women from the United Kingdom. Am J Obstet Gynecol 189, 1343-1349.

31. Xia Y, Hill KE, Li P, et al. (2010) Optimization of selenoprotein P and other plasma selenium biomarkers for the assessment of the selenium nutritional requirement: a placebo-controlled, double-blind study of selenomethionine supplementation in selenium-deficient Chinese subjects. Am J Clin Nutr 92, 525-531.

32. Haghiac M, Basu S, Presley L, et al. (2014) Patterns of adiponectin expression in term pregnancy: impact of obesity. J Clin Endocrinol Metab 99, 3427-3434.

33. Catalano PM, Tyzbir ED, Roman NM, et al. (1991) Longitudinal changes in insulin release and insulin resistance in nonobese pregnant women. Am J Obstet Gynecol 165, 1667-1672.

34. Rayman MP (1997) Dietary selenium: time to act. BMJ 314 , 387-388.

35. Misu H, Ishikura K, Kurita S, et al. (2012) Inverse correlation between serum levels of selenoprotein $\mathrm{P}$ and adiponectin in patients with type 2 diabetes. PLOS ONE 7, e34952.

36. Chung SS, Kim M, Youn BS, et al. (2009) Glutathione peroxidase 3 mediates the antioxidant effect of peroxisome proliferator-activated receptor gamma in human skeletal muscle cells. Mol Cell Biol 29, 20-30.

37. Rayman MP, Wijnen H, Vader H, et al. (2011) Maternal selenium status during early gestation and risk for preterm birth. CMAJ 183, 549-555.

38. Molnar J, Garamvolgyi Z, Herold M, et al. (2008) Serum selenium concentrations correlate significantly with inflammatory biomarker high-sensitive CRP levels in Hungarian gestational diabetic and healthy pregnant women at mid-pregnancy. Biol Trace Elem Res 121, 16-22.

39. Negro R, Greco G, Mangieri T, et al. (2007) The influence of selenium supplementation on postpartum thyroid status in pregnant women with thyroid peroxidase autoantibodies. J Clin Endocrinol Metab 92, 1263-1268. 